\title{
Influência da Incorporação de Dados de Progênies na Classificação de Touros da Raça Nelore
}

\author{
Analía del Valle Garnero1, 2, Mauricio Barros Fernandes ${ }^{1,2}$, Luiz Fernando Camilo Figueiredo1, \\ Raysildo Barbosa Lôbo ${ }^{1,3}$
}

RESUMO - No Programa de Melhoramento Genético da Raça Nelore (PMGRN) o índice Mérito Genético Total (MGT) reúne em uma única informação as diferentes predições do valor genético de cinco características. O índice considera as diferenças esperadas na progênie (DEP) para efeito materno sobre o peso aos 120 dias de idade; efeito direto do peso aos 120, 365 e 450 dias de idade; e efeito direto do perímetro escrotal aos 365 e 450 dias de idade. O objetivo deste trabalho foi identificar e descrever a mudança que ocorre no MGT de touros ao incorporar informações provenientes de seus descendentes. Em 29.769 observações, foram selecionados 12 touros que geraram três arquivos contendo informações do animal, do animal mais as da sua progênie e do animal mais as da sua progênie e de seus netos. Realizaram-se análises unicaráter utilizando-se o software MTDFREML (Multiple Trait Derivative Free Restricted Maximum Likelikood). O modelo para as características de peso até um ano de idade considerou, como fixos, os efeitos de grupos de contemporâneos e classe de idade da vaca ao parto e, como aleatórios, os efeitos genéticos direto, materno e de ambiente permanente da vaca. Para as características de perímetro escrotal e de peso após um ano de idade, empregaram-se os mesmos efeitos fixos e, como aleatório, o efeito genético direto. Quando se incorporaram as informações das progênies, as mudanças na classificação foram, no máximo, de duas posições. Quando as informações dos netos foram adicionadas, as posições dos touros na classificação permaneceram inalteradas.

Palavra-chave: classificação, índice de seleção, Nelore, parâmetros genéticos, valor genético

\section{Effect of Progeny Data on the Ranking of Nellore Bulls}

\begin{abstract}
In the Nellore Breed Genetic Improvement Program (PMGRN) the breeding value predictions of five different traits are put together in a single number named Total Genetic Merit (MGT). This number is calculated from the Expected Progeny Differences (EPDs) of: weight at 120 days of age - maternal; weight at 120, 365 and 450 days of age direct; and scrotal circumference at 365 and 450 days of age direct. The aim of this study was to identify and to describe the change in the MGT of bulls when information on their progenies are used. Twelve sires were selected, from a total database of 29,759 records, generating three files containing: individual performance; individual performance plus progeny performance; and individual performance plus progeny and grandprogeny performance. Single analyses were performed for all traits utilizing MTDFREML software (Multiple Traits Derivative Free Restricted Maximum Likelihood). The fixed effects (contemporary groups and cow age at calving) and the random effects (direct, maternal and permanent environmental) were included in the model for growth traits up to one year of age. The same fixed effects were included in the model for scrotal circumference traits and for body weight taken after one year of age and the direct effects as random effects. When the offspring data were added, ranking changes were achieved between classes at most in two positions. When grandprogerny were added, no changes were found.
\end{abstract}

Key Words: genetic parameters, genetic value, selection index, ranking, Nellore

\section{Introdução}

O valor econômico de um animal como reprodutor não é determinado por uma característica isolada, mas sim pelo conjunto das características de maior interesse econômico. Desde a introdução do índice de seleção em suínos, em 1943, por Hazel, várias pesquisas foram realizadas em diferentes espécies. Em gado de corte, muitos estudos foram realizados mostrando as vantagens da utilização de índice de seleção (Lindholm \&
Stonaker, 1957; Lehmann et al., 1961; Vesely \& Robison, 1971; Dickerson et al., 1974).

De modo geral, a seleção baseada em mais de um caráter pode ser obtida com base em um índice que envolva as $\mathrm{n}$ características, atribuindo pesos ou valores diferentes a cada uma delas. Os pesos de cada característica, em bovinos de corte, podem ser estimados pelo conhecimento de suas (co)variâncias, que se modificam conforme a fração de animais selecionados (Dickerson et al., 1974).

\footnotetext{
${ }^{1}$ Departamento de Genética - Bloco C - Faculdade de Medicina de Ribeirão Preto - USP - Av. Bandeirantes, 3900 - $14049-900$. Ribeirão Preto, SP. E.mail: agarnero@rgm.fmrp.usp.br; rayblobo@genbov.fmrp.usp.br; Ifernando@anep.org.br; mfb@genbov.fmrp.usp.br 2 Bolsista CAPES.

${ }^{3}$ Bolsista I-A do CNPq
} 
A eficiência do índice de seleção apresenta relação direta com a precisão das estimativas de parâmetros genéticos das características que o compõem, e, quanto maior for, melhor será a eficiência do índice (Vesely \& Robison, 1971). Nesse sentido, Sivanadian \& Smith (1997), estudando o efeito da adição de características ao índice, concluíram que, quando os parâmetros são bem estimados, todas as caraterísticas devem ser incorporadas ao índice, sem reduzir a reposta à seleção.

Alguns índices compreendem características de crescimento, pesos ou ganhos, outros incorporam características de conformação, mas na maioria o peso ao desmame apresenta o maior valor ou peso dentro do índice, quando comparado com as outras características (Lindholm \& Stonaker, 1957; Lehmann et al., 1961; Vesely \& Robison, 1971; Pons et al., 1990). Segundo alguns autores (Lindholm \& Stonaker, 1957; Vesely \& Robison, 1971), do ponto de vista prático da seleção, a característica peso ao desmame pode ser considerada, por si só, o melhor critério, além de ser, economicamente, a mais interessante.

No momento, todos os programas de melhoramento praticados no Brasil utilizam um índice, geralmente baseado em agregados genotípicos, alguns levando em conta características de crescimento e sexuais, e outros incorporando características de conformação (CFM, 2000; Lôbo et al., 2000; Paint, 2000).

O Mérito Genético Total (MGT) é o índice utilizado pelo Programa de Melhoramento Genético da Raça Nelore (PMGRN) que reúne em uma única informação as diferenças esperadas na progênie (DEP) dos pesos padronizados aos 120, 365 e 450 dias (P120, P365, P450) e dos perímetros escrotais aos 365 e 450 dias (PE365, PE450) (Lôbo et al., 1999). Esse índice vem sendo utilizado desde 1995, a partir daí, algumas modificações têm sido realizadas buscando maximizar o progresso genético.

As DEPs são predições da capacidade de transmissão obtidas com quantidades de informação que variam de um indivíduo para outro. Dessa forma, a segurança com a qual as DEPs são estimadas também varia. Esta segurança recebe vários nomes, sendo o mais comum, acurácia (Bergmann, 2001).

Em grandes populações, o cálculo da acurácia é mais demorado computacionalmente. Por isto, torna-se importante determinar o grau de confiabilidade que se tem no valor genético, calculado a partir de um modelo animal, de um animal sem filhos, com filhos, com filhos e com netos, sem necessidade de calcular a acurácia.

O principal objetivo deste estudo foi identificar e descrever a mudança que ocorre no MGT de machos jovens e na classificação dos mesmos, quando são incorporadas as informações provenientes de seus descendentes.

\section{Material e Métodos}

As análises foram feitas com um arquivo de 29.769 observações referentes a animais da raça Nelore. Foram estimados os parâmetros genéticos e as diferenças esperadas na progênie (DEP) para as características: pesos padronizados aos 120 (P120), 365 (P365) e 450 (P450) dias de idade e perímetros escrotais aos 365 (PE365) e 450 (PE450) dias de idade.

Para o ajuste dos pesos às idades-padrão, foi necessária a obtenção, primeiramente, do ganho médio diário (GMD), que é a razão da diferença entre os pesos posterior e anterior à idade-padrão pelo número de dias contidos no período compreendido entre as duas pesagens.

As análises estatísticas básicas foram realizadas utilizando-se o software Statistical Analysis System (SAS Institute, 1996) e a estimação dos parâmetros genéticos e fenotípicos e dos valores genéticos, empregando-se o software MTDFREML (Multiple Trait Derivative Free Restricted Maximum Likelikood), descrito por Boldman et al. (1995).

Foram realizadas análises unicaráter de todas as características, utilizando-se diferentes modelos, conforme o caso. Para a análise das características prédesmama, utilizou-se o modelo 1 que incluiu, como fixos, os efeitos de grupos de contemporâneos e classe de idade da vaca ao parto e, como aleatórios, os efeitos genéticos direto, materno e de ambiente permanente da vaca.

Para as características pós-desmama, utilizaramse os modelos 2 e 3 , o primeiro incluiu, como fixos, os efeitos de grupo de contemporâneos e classe de idade da vaca ao parto e, como aleatórios, os efeitos genéticos direto e materno; o segundo modelo incluiu os mesmos efeitos fixos, mas como aleatório somente o efeito direto.

Em termos matriciais, os modelos podem ser descritos como:

$$
\begin{array}{ll}
Y=X b+Z_{1} a+Z_{2} m+Z_{3} p+e & (\text { modelo } 1) \\
Y=X b+Z_{1} a+Z_{2} m+e & (\text { modelo } 2) \\
Y=X b+Z_{1} a+e & (\text { modelo } 3)
\end{array}
$$


em que: $\mathrm{Y}=$ vetor das observações de cada característica; $X=$ matriz de incidência dos efeitos fixos; $\mathrm{b}=$ vetor dos efeitos fixos; $\mathrm{Z}_{1}=$ matriz de incidência do efeito genético direto de cada animal; $a=$ vetor de efeitos aleatórios genéticos diretos; $Z_{2}=$ matriz de incidência do efeito genético materno de cada animal; $\mathrm{m}=$ vetor de efeitos aleatórios genéticos maternos; $\mathrm{Z}_{3}=$ matriz de incidência do efeito de ambiente permanente; $\mathrm{p}=$ vetor de efeitos aleatórios de ambiente permanente; $\mathrm{e}=$ vetor de efeitos aleatórios residuais.

Os grupos de contemporâneos (GC) foram constituídos por animais nascidos na mesma fazenda, na mesma época do ano, do mesmo sexo e no mesmo regime alimentar (pasto ou suplementação). Para a formação dos grupos de contemporâneos aos 120 dias (GC120), foram considerados quatro trimestres de nascimentos (de janeiro a março, de abril a junho, de julho a setembro e de outubro a dezembro). Entretanto, para os grupos de contemporâneos aos 365 e 450 dias (GC365, GC450), utilizaram-se os semestres de nascimento de janeiro a junho e de julho a dezembro.

Dentro do arquivo total de informações ( $n=29769)$, foram estudados 12 touros quanto à sensibilidade de seu MGT à incorporação de dados de seus descendentes. Desse arquivo ordenado cronologicamente, foram escolhidos os primeiros 12 touros com um mínimo de 20 filhos (Tabela 1). A partir desse arquivo total foram gerados três arquivos. No primeiro, foram apagadas as informações dos filhos e netos desses 12 touros ( $\mathrm{P}, \mathrm{n}=29142)$, no segundo arquivo foram acrescentadas as informações referentes aos filhos (F1, $\mathrm{n}=29660)$ e, finalmente, no terceiro, as informações dos netos ( $F 2, n=29769)$.

Atualmente, o cálculo do MGT para machos leva em conta as seguintes ponderações: $10 \%$ da DEP para efeito materno no peso aos 120 dias de idade (DEPMP120); 20\% da DEP para efeito direto no peso aos 120 dias de idade (DEPDP120); 20\% da DEP para efeito direto no peso aos 365 e 450 dias de idade (DEPDP365 e DEPDP450); e 15\% da DEP para efeito direto no perímetro escrotal aos 365 e 450 dias de idade (DEPPE365 e DEPPE450).

Como as DEPs são expressas em unidades diferentes de acordo com a característica, as mesmas devem ser padronizadas em unidades equivalentes previamente ao cálculo do MGT. Dessa forma, cada DEP foi expressa em unidades de desvio-padrão genético (DPG), obtido de acordo com cada tipo de arquivo analisado (Tabela 2).

R. Bras. Zootec., v.31, n.2, p.918-923, 2002 (suplemento)
Tabela 1 - Filiação e freqüência de filhos dos touros selecionados

Table 1 - Parents and number of progeny by seleted bull

\begin{tabular}{lrrc}
\hline $\begin{array}{l}\text { Touro } \\
\text { Bull }\end{array}$ & Pai & $\begin{array}{c}\text { Mãe } \\
\text { Dam }\end{array}$ & $\begin{array}{c}\text { No filhos } \\
N^{o} \text { progeny }\end{array}$ \\
\hline A & 3237 & 7314 & 43 \\
B & 3237 & 6530 & 88 \\
C & 3237 & 7258 & 30 \\
D & 3250 & 7113 & 65 \\
E & 3237 & 14.712 & 28 \\
F & 7263 & 14.674 & 61 \\
G & 3237 & 15.047 & 34 \\
H & 15.478 & 15.047 & 51 \\
I & 7498 & 15.517 & 37 \\
J & 3260 & 14.528 & 27 \\
K & 2993 & 14.048 & 85 \\
L & 15.521 & 15.763 & 20 \\
\hline
\end{tabular}

Tabela 2 - Desvio-padrão genético (DPG), segundo a característica e o arquivo

Table 2 - Genetic standard deviation (DPG), for traits and files

\begin{tabular}{lccc}
\hline & \multicolumn{3}{c}{$\begin{array}{c}\text { Arquivo } \\
\text { Files }\end{array}$} \\
\cline { 2 - 4 } & $\mathrm{P}^{1}$ & $\mathrm{~F} 1^{1}$ & $\mathrm{~F} 2$ \\
\hline DEPMP120 $^{2}$ & 2,03 & 2,03 & 2,02 \\
DEPDP120 $^{2}$ & 3,41 & 3,23 & 3,48 \\
DEPDP365 $^{2}$ & 6,21 & 6,21 & 6,20 \\
DEPDP450 $^{2}$ & 8,54 & 8,50 & 8,80 \\
DEPDPE365 $^{2}$ & 6,00 & 6,00 & 6,00 \\
DEPDPE450 $^{2}$ & 8,20 & 8,20 & 8,14 \\
\hline
\end{tabular}

${ }^{1} \mathrm{P}$ : parentais e a sua genealogia; F1: filhos; F2: netos.

2 DEPMP120: diferença esperada na progênie para efeito materno para peso aos 120 dias de idade; DEPDP"dias": diferença esperada na progênie para efeito direto para peso aos 120, 365 ou 450 dias de idade; DEPPE"dias": diferença esperada na progênie para efeito direto para perímetro escrotal aos 365 e 450 dias de idade

${ }^{1} \mathrm{P}$ : individual performance; F1: individual performance plus progeny; F2: individual performance plus progeny and grandprogeny.

2 DEPMP120: expected progeny difference for maternal ability; DEPDP"days": expected progeny difference for growth at 120, 365, 450 days old; DEPPE"days": expected progeny difference for scrotal circumference at 365 and 450 days old.

\section{Resultados e Discussão}

As médias (Tabela 3) dos pesos padronizados e os perímetros escrotais estão de acordo com os citados na literatura para a raça Nelore e com os valores dos programas de melhoramento que, atualmente, estão sendo conduzidos no Brasil.

Nas variáveis P120 e P365 (Tabela 4), verificaram-se valores de herdabilidade direta de mediana magnitude, dentro dos valores esperados para rebanhos da raça Nelore no Brasil (Eler et al., 1995; Mercadante 
Tabela 3 - Número de observações (n), médias gerais $(m)$, coeficiente de variação (cv), e valores máximos (max) e mínimos (min) das características analisadas

Table 3 - Number of observations ( $n$ ), means, coefficient of variation (cv), and minimum (min) and maximum (max) values for the traits studied

\begin{tabular}{|c|c|c|c|c|}
\hline \multirow[b]{2}{*}{$\begin{array}{l}\text { Característica }{ }^{2} \\
\text { Trait }\end{array}$} & & \multicolumn{3}{|c|}{$\begin{array}{l}\text { Arquivo } \\
\text { Files }\end{array}$} \\
\hline & & $\mathrm{P}^{1}$ & $\mathrm{~F} 1^{1}$ & $\mathrm{~F} 2^{1}$ \\
\hline \multirow[t]{5}{*}{ P120(kg) } & $\mathrm{n}$ & 29009 & 29522 & 29631 \\
\hline & $\mathrm{m}$ & 121,08 & 121,27 & 121,29 \\
\hline & $\mathrm{cV}$ & 15,51 & 15,48 & 15,49 \\
\hline & $\max$ & 207 & 207 & 207 \\
\hline & $\min$ & 55 & 55 & 55 \\
\hline \multirow[t]{5}{*}{ P365 (kg.) } & $\mathrm{n}$ & 21755 & 22220 & 22306 \\
\hline & $\mathrm{m}$ & 225,56 & 225,59 & 225,63 \\
\hline & $\mathrm{cV}$ & 17,29 & 17,27 & 17,29 \\
\hline & $\max$ & 460 & 460 & 460 \\
\hline & $\min$ & 99 & 99 & 99 \\
\hline \multirow[t]{5}{*}{$\mathrm{P} 450(\mathrm{~kg})$} & $\mathrm{n}$ & 18051 & 18492 & 18548 \\
\hline & $\mathrm{m}$ & 263,68 & 263,70 & 268,70 \\
\hline & $\mathrm{cV}$ & 17,37 & 17,35 & 17,34 \\
\hline & $\max$ & 546 & 546 & 546 \\
\hline & $\min$ & 122 & 122 & 122 \\
\hline \multirow[t]{5}{*}{$\mathrm{PE} 365(\mathrm{~mm})$} & $\mathrm{n}$ & 5531 & 5644 & 5668 \\
\hline & $\mathrm{m}$ & 196,35 & 196,32 & 196,35 \\
\hline & $\mathrm{cV}$ & 10,76 & 10,77 & 10,76 \\
\hline & $\max$ & 311 & 311 & 311 \\
\hline & $\min$ & 131 & 131 & 131 \\
\hline \multirow[t]{5}{*}{$\mathrm{PE} 450(\mathrm{~mm})$} & $\mathrm{n}$ & 5916 & 6120 & 6150 \\
\hline & $\mathrm{m}$ & 229,05 & 228,92 & 228,85 \\
\hline & $\mathrm{cV}$ & 11,96 & 11,93 & 11,93 \\
\hline & $\max$ & 352 & 352 & 352 \\
\hline & $\min$ & 150 & 150 & 150 \\
\hline
\end{tabular}

${ }_{1} \mathrm{P}$ : parentais e a sua genealogia; F1: filhos; F2: netos.

2 P"dias": peso padronizado aos 120, 365 e 450 dias, PE"dias": perímetro escrotal padronizado aos 365 e 450 dias.

$1 \mathrm{P}$ : individual performance; F1: individual performance plus progeny; F2: individual performance plus progeny and grandprogeny.

2 P"days"= standardized weight at 120,365 and 450 days old. PE"days": standardized scrotal circumference at 365 and 450 days of age. et al., 1997; Lôbo et al., 1999; Garnero, 1999). No entanto, as estimativas de herdabilidade materna para o P365 apresentaram valor acima do esperado e do citado na bibliografia, observando-se a influência materna ainda aos doze meses de idade.

Para o P450 obteve-se coeficiente de herdabilidade superior, quando comparado com as outras características de crescimento (Tabela 4), mas levemente inferior ao reportado por Siqueira (2000), concordando o fato mencionado autor de que o P450 é um bom critério de seleção, visando à redução da idade de avaliação genética nos programas de melhoramento em gado de corte.

Os valores de herdabilidade estimados para os perímetros escrotais padronizados a diferentes idades (Tabela 5) foram levemente maiores que os encontrados na literatura para a raça Nelore (Lôbo et al., 1999; Garnero, 1999; Siqueira, 2000).

Os componentes de variâncias para cada característica apresentaram valores semelhantes nos três arquivos estudados (P, F1 e F2) e, em conseqüência, os coeficientes de herdabilidade direta e materna também, assim como a contribuição do ambiente permanente da vaca e do resíduo.

Observa-se, na Tabela 6, que as maiores mudanças no MGT aconteceram quando informações dos filhos foram incorporadas ao arquivo $\mathrm{P}$ (dos pais). No entanto, quando as informações referentes aos netos foram incorporadas, não se observaram mudanças sensíveis no valor do MGT previamente estimados.

Assim, oito dos doze touros estudados diminuíram seus valores para a DEPDP365, enquanto seis touros

Tabela 4 - Componentes de variância ${ }^{3}$ e valores de herdabilidade ${ }^{3}$ para pesos padronizados Table 4 - Variance ${ }^{3}$ components and heritability ${ }^{3}$ for standardized body weights

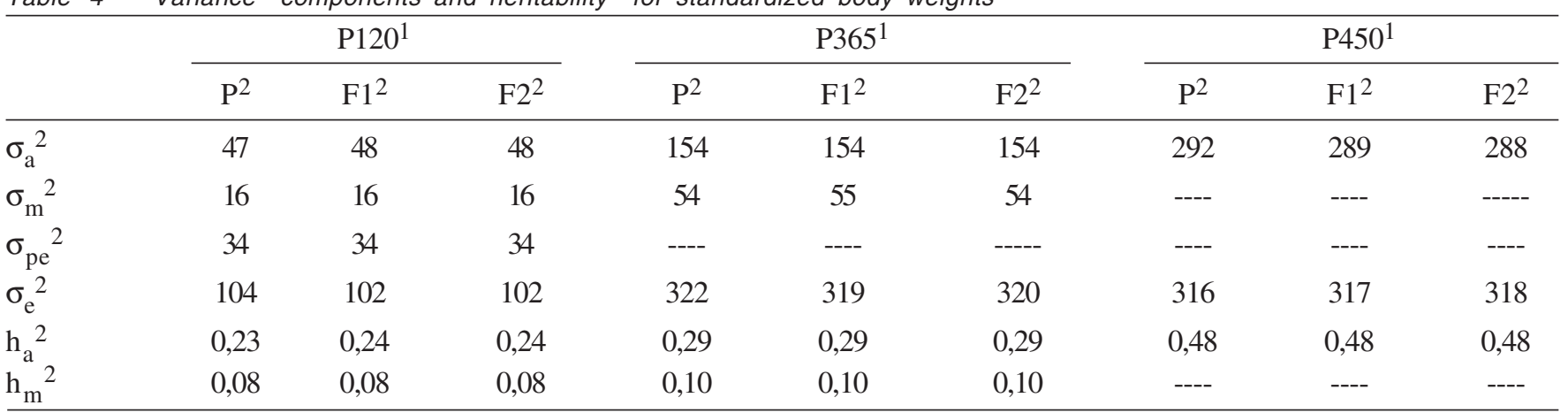

${ }_{1}$ P"dias"= peso padronizado a uma determinada idade em dias ( $P$ "days"= standardized weight).

$2 \mathrm{P}$ : parentais e a sua genealogia; F1: filhos; F2: netos ( $P$ : individual performance; F1: individual performance plus progeny; F2 individual performance plus progeny and grandprogeny).

${ }^{3} \sigma^{2 a}$ : variância aditiva direta, $\sigma^{2} \mathrm{~m}$ : variância aditiva materna, $\sigma^{2}$ pe: variância de ambiente permanente, $\sigma^{2} \mathrm{e}$ : variância do erro, $h^{2} \mathrm{a}$ : herdabilidade direta, $h^{2} \mathrm{~m}$ : herdabilidade materna $\left(\sigma_{a}^{2}=\right.$ direct additive genetic variance; $\sigma_{m}{ }^{2}=$ additive maternal variance; $\sigma_{p e}{ }^{2}=$ permanent environmental variance; $\sigma_{e}{ }^{2}=$ residual variance $h_{a}^{2}=$ direct heritability, $h_{m}{ }^{2}=$ additive maternal heritability).

R. Bras. Zootec., v.31, n.2, p.918-923, 2002 (suplemento) 
Tabela 5 - Componentes de variância ${ }^{3}$ e herdabilidade ${ }^{3}$ para perímetro escrotal

Table 5 - Variance ${ }^{3}$ components and heritability ${ }^{3}$ for scrotal circumference

\begin{tabular}{|c|c|c|c|c|c|c|}
\hline & \multicolumn{3}{|c|}{ PE365 ${ }^{1}$} & \multicolumn{3}{|c|}{$\mathrm{PE} 450^{1}$} \\
\hline & $\mathrm{P}^{2}$ & $\mathrm{~F} 1^{2}$ & $\mathrm{~F} 2^{2}$ & $\mathrm{P}^{2}$ & $\mathrm{~F} 1^{2}$ & $\mathrm{~F} 2^{2}$ \\
\hline$\sigma_{\mathrm{a}}^{2}$ & 144 & 144 & 143 & 267 & 267 & 265 \\
\hline$\sigma_{\mathrm{e}}^{2}$ & 132 & 132 & 133 & 232 & 232 & 233 \\
\hline $\mathrm{h}_{\mathrm{a}}{ }^{2}$ & 0,52 & 0,52 & 0,52 & 0,53 & 0,53 & 0,53 \\
\hline \multicolumn{7}{|c|}{ 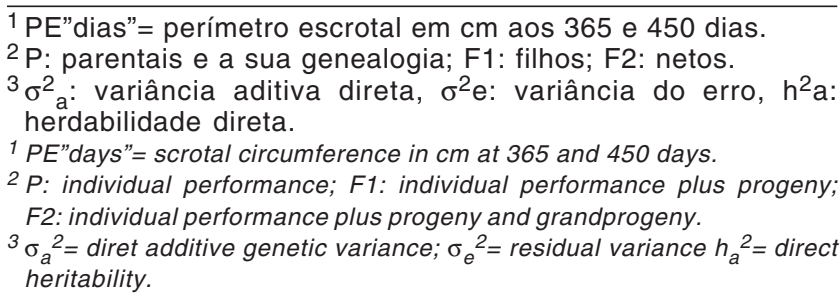 } \\
\hline
\end{tabular}

mostraram o mesmo efeito para a DEPDP450, quando foram incorporadas as informações dos filhos. De forma geral, as DEPs estimadas para o efeito materno aos 120 dias e os perímetros escrotais, a partir das informações próprias dos touros $(\mathrm{P})$, não sofreram grandes mudanças quando foram incorporadas as informações dos filhos e netos.

As acurácias estimadas a partir do arquivo $\mathrm{P}$ variaram de 0,62 a 0,81 para as DEPs diretas e 0,46 a 0,69 para as maternas. Quando estimadas a partir dos arqui- vos F1 e F2, variaram de 0,85 a 0,95 para as DEPs diretas e de 0,46 a 0,70 para as maternas, à exceção do touro $\mathrm{H}$, que apresentou valores inferiores, variando as acurácias de 0,42 a 0,50 e 0,38 a 0,41 para direta e materna, respectivamente, quando estimadas a partir do arquivo P. Quando acrescentadas as informações dos filhos e netos, os valores alteram-se para 0,53 a 0,63 para as DEPs diretas e 0,39 a 0,41 para as maternas.

Em virtude dessas mínimas mudanças observadas nos valores das DEPs e, conseqüentemente, nos valores dos MGTs, quando se incorporam novas informações, e dos valores de acurácia obtidos, pode-se concluir que a genealogia desses animais é suficientemente numerosa e consistente, não permitindo que os dados provenientes dos filhos e dos netos alterem de maneira relevante a classificação dos touros analisados, conferindo confiabilidade ao valor genético dos touros, mesmo que não tenham descendentes, facilitando a escolha de animais em idades mais jovens, encurtando o intervalo de gerações, acelerando o progresso genético.

As maiores mudanças nos valores de MGT (Tabelas 6), como era esperado, já que o valor do índice depende dos valores das DEPs, aconteceram quando informações dos filhos foram incorporadas ao arquivo com informações dos próprios touros.

Como pode-se observar na Tabela 6, quando são incorporadas as informações dos filhos, as mudanças

Tabela 6 - Classificação dos touros segundo o MGT dos arquivos $\mathrm{P}$ (que contem informações de pesagens dos próprios touros e a sua genealogia), F1 (informações próprias mais da progênie) e F2 (informações próprias mais da progênie e netos)

Table 6 - Ranking of bulls according to MGT for file $P$ (individual and ancestry performance), F1(individual, ancestry and offspring performance) and F2 (individual, ancestry, offspring and grandprogeny performance)

\begin{tabular}{|c|c|c|c|c|c|c|}
\hline \multirow[b]{3}{*}{$\begin{array}{l}\text { Classificação } \\
\text { Ranking }\end{array}$} & \multicolumn{6}{|c|}{$\begin{array}{l}\text { Arquivo } \\
\text { Files }\end{array}$} \\
\hline & \multicolumn{2}{|c|}{$\mathrm{P}$} & \multicolumn{2}{|c|}{$\mathrm{F} 1$} & \multicolumn{2}{|c|}{$\mathrm{F} 2$} \\
\hline & $\begin{array}{c}\text { Touro } \\
\text { Bull }\end{array}$ & $\mathrm{MGT}^{1}$ & $\begin{array}{c}\text { Touro } \\
\text { Bull }\end{array}$ & $\mathrm{MGT}^{1}$ & $\begin{array}{c}\text { Touro } \\
\text { Bull }\end{array}$ & $\mathrm{MGT}^{1}$ \\
\hline 1 & B & 1,16 & B & 1,22 & B & 1,18 \\
\hline 2 & $\mathrm{G}$ & 1,00 & $\mathrm{G}$ & 1,20 & $\mathrm{G}$ & 1,16 \\
\hline 3 & A & 0,94 & $\mathrm{C}$ & 1,01 & $\mathrm{C}$ & 0,99 \\
\hline 4 & $\mathrm{C}$ & 0,89 & A & 0,76 & A & 0,74 \\
\hline 5 & $\mathrm{~F}$ & 0,88 & $\mathrm{~F}$ & 0,55 & $\mathrm{~F}$ & 0,54 \\
\hline 6 & D & 0,35 & $\mathrm{E}$ & 0,32 & $\mathrm{E}$ & 0,31 \\
\hline 7 & $\mathrm{~J}$ & 0,28 & $\mathrm{H}$ & 0,28 & $\mathrm{H}$ & 0,26 \\
\hline 8 & $\mathrm{E}$ & 0,28 & $\mathrm{D}$ & 0,19 & $\mathrm{D}$ & 0,18 \\
\hline 9 & $\mathrm{H}$ & 0,18 & $\mathrm{~J}$ & $-0,02$ & $\mathrm{~J}$ & $-0,02$ \\
\hline 10 & I & 0,08 & $\mathrm{~L}$ & $-0,05$ & $\mathrm{~L}$ & $-0,06$ \\
\hline 11 & $\mathrm{~L}$ & $-0,02$ & $\mathrm{~K}$ & $-0,22$ & $\mathrm{~K}$ & $-0,23$ \\
\hline 12 & $\mathrm{~K}$ & $-0,21$ & I & $-0,47$ & I & $-0,47$ \\
\hline
\end{tabular}

${ }^{1}$ MGT: Índice Mérito Genético Total.

MGT: Total Genetic Merit Index.

\section{R. Bras. Zootec., v.31, n.2, p.918-923, 2002 (suplemento)}


na classificação são, no máximo, de apenas duas posições. Entretanto, quando as informações dos netos são tomadas em conta as posições na classificação de cada touro nos arquivos F1 e F2, permanecem inalteradas.

No futuro, seria interessante fazer as análises com o mesmo arquivo de dados, mas analisando-se primeiramente os touros sem genealogia, logo incorporando os pais, os avós e, assim, sucessivamente, com a finalidade de se determinar qual é o verdadeiro valor da genealogia sobre os valores genéticos dos animais.

\section{Conclusões}

Para as características analisadas, os resultados confirmam a importância da genealogia e, em menor magnitude, a influência das informações do desempenho da progênie, quando se prediz o valor genético dos reprodutores. As informações provenientes dos netos, de acordo com o presente trabalho, não influenciaram os valores preditos dos reprodutores.

\section{Agradecimento}

Ao A. Sist. Luiz A. F. Bezerra, ao Prof. Dr. Henrique Nunes de Oliveira, às entidades financeiras FAEPA, FAPESP, FINEP/BID, CNPq/RHAE, ANCP, CAPES e aos criadores do PMGRN.

\section{Literatura Citada}

BERGMANN, J.A.G. Avaliação genética. In: PEREIRA, J.C.C. (Ed.) Melhoramento genético aplicado à produção animal. 3.ed. Belo Horizonte: 2001. p.502-515.

BOLDMAN, K.G.; KRIESE, L.A.; Van VLECK, L.D. et al. A manual for use for MTDFREML- a set of programs to obtain of variance and covariances [DRAF]. Washington: Department of Agriculture/ Agricultural Research Service. 1995.

CFM. Sumário de Touros Nelore 2000. Publicação da AgroPecuária CFM. - São José do Rio Preto: Agro-Pecuária CFM, 2000, 60p.

ELER, J.P.; Van VLECK, L.D.; FERRAZ, J.B.S. et al. Estimation of variances due to direct and maternal effects for growth traits of Nelore cattle. Journal of Animal Science, v.77, p.3253-3258, 1995.

GARNERO, A. del V. Comparação de critérios de seleção em gado de corte visando precocidade de crescimento. Ribeirão Preto: Universidade São Paulo, 1999. 85p. Dissertação (Mestrado em Ciências) - Universidade São Paulo, 1999.

HAZEL, L.N. The genetic basis for constructing selection indexes. Genetics, v.28, p.476-490, 1943.
LEHMANN, R.P.; GAINES, J.A.; CARTER, R.C. et al. Selection indexes for weanling traits in beef calves. Journal of Animal Science, v.20, n.1, p.53-57, 1961.

LINDHOLM, H.B.; STONAKER, H.H. Economic importance of traits and selection indexes for beef cattle. Journal of Animal Science, v.16, n.3 p.998-1006, 1957.

LÔBO, R.B.; BEZERRA, L.A.F.; OLIVEIRA, H.N. Avaliação genética de animais jovens, touros e matrizes. Ribeirão Preto: GEMAC - Departamento de Genética-FMRP - USP, 1999. 82p.

LÔBO, R.B.; BEZERRA, L.A.F.; OLIVEIRA, H.N. et al. Avaliação genética de animais jovens, touros e matrizes. Ribeirão Preto: GEMAC - Departamento de Genética FMRP - USP, 2000. 90p.

MERCADANTE, M.E.Z.; LÔBO, R.B. Estimativas de (co)variância e parâmetro genético dos efeitos direto e materno de características de crescimento de fêmeas de um rebanho Nelore. Revista Brasileira de Zootecnia, v.26, n.6, p.1124-1133, 1997.

PAINT. Sumário PAINT 2000. 23p.

PONS, S.B.; MILAGRES, J.C.; REGAZZI, A.J. Efeitos de fatores genéticos e de ambiente sobre o crescimento e o escore de conformação em bovinos Hereford no Rio Grande do Sul III. Peso e escore de conformação ao sobreano. Revista Brasileira de Zootecnia, v.19, n.2, p.77-82, 1990.

SAS INSTITUTE Inc. SAS/STAT ${ }^{T M}$. SAS user's guide for windows environment. 6.08 ed. Cary, SAS Institute Inc., 1996.

SINAVANADIAN, B.; SMITH, C. The effect of adding further traits in index selection. Journal of Animal Science, v.75, p.2016-2023, 1997.

SIQUEIRA, R.L.P.G. Análise da variabilidade genética aditiva das características de crescimento na raça Nelore. Jaboticabal: Universidade Estadual Paulista, 2000. 84p. Dissertação (Mestrado em Zootecnia) - Universidade Estadual Paulista, 2000.

VESELY, J.A.; ROBISON, O.W. Conventional selection indexes for birth and weaning traits in beef calves. Journal of Animal Science, v.33, n.3, p.537-541, 1971. 